Tropical Journal of Pharmaceutical Research February 2013; 12 (1): 13-18

ISSN: $1596-5996$ (print); 1596-9827 (electronic)

(c) Pharmacotherapy Group, Faculty of Pharmacy, University of Benin, Benin City, 300001 Nigeria.

All rights reserved.

Available online at http://www.tjpr.org

Original Research Article

http://dx.doi.org/10.4314/tjpr.v12i1.3

\title{
Formulation and Evaluation of Bupivacaine-Loaded Glutaraldehyde-Crosslinked High Molecular Weight Chitosan Microspheres
}

\author{
Murugesh Shivashankar ${ }^{1}$ and Badal Kumar Manda ${ }^{2^{*}}$ \\ ${ }^{1}$ Pharmaceutical Chemistry Division, ${ }^{2}$ Environmental and Analytical Chemistry Division, School of Advanced Sciences, VIT \\ University, Vellore 632014, Tamilnadu, India
}

*For correspondence: E-mail: badalmandal@vit.ac.in; Tel: +91 (0416) 2202339

Received: 22 November 2012

Revised accepted: 15 January 2012

\begin{abstract}
Purpose: To develop a chitosan microsphere carrier system of bupivacaine for buccal administration. Methods: Chitosan microspheres loaded with bupivacaine were prepared by emulsification technique based on glutaraldehyde cross-linking and drug-loaded chitosan microsphere were coated with polyglycolic acid (PGA) film The formulated microspheres were characterized by $x$-ray diffraction (XRD), differential scanning calorimetry (DSC), scanning electron microscopy (SEM) and in vitro release was performed in $\mathrm{pH} 7.4$ phosphate buffer both in the presence and in absence of lysozyme.

Results: Encapsulation yield was $83.1 \%$. SEM studies indicate that the microspheres were spherical and had a relatively smooth surface. XRD and DSC data indicate that there was no interaction between the drug and polymer. In vitro results show that in the presence of lysozyme, $39 \%$ of the drug was released from the microspheres after 4.5 days while maximum drug release (42.5\%) was achieved on day 11. This compares with $31 \%$ drug release on $4.5^{\text {th }}$ day and $38 \%$ on $11^{\text {th }}$ day; maximum drug release occurred on day 11 in the absence of lysozyme.

Conclusion: It is evident from this study that microspheres can potentially be used for controlled release of of bupivacaine for the management of dental pain in the buccal cavity.
\end{abstract}

Keywords: Cross-linked chitosan, Microsphere, In vitro release, Oral drug delivery, Bupivacaine, Local anesthesia.

Tropical Journal of Pharmaceutical Research is indexed by Science Citation Index (SciSearch), Scopus, International Pharmaceutical Abstract, Chemical Abstracts, Embase, Index Copernicus, EBSCO, African Index Medicus, JournalSeek, Journal Citation Reports/Science Edition, Directory of Open Access Journals (DOAJ), African Journal Online, Bioline International, Open-J-Gate and Pharmacy Abstracts

\section{INTRODUCTION}

Compared to general anesthesia with opioidbased preoperative pain management regional anesthesia can provide benefits of superior pain control, improved patient satisfaction, decreased stress response to surgery reduced operative and post operative blood loss. Local anesthesia (LA) is valued for the ability to prevent membrane depolarization of nerve cells. LA prevents depolarization of nerve cells by binding to cell membrane sodium channels and inhibiting the passage of sodium ions.
Bupivacaine has been widely used for local or regional anesthesia after surgery because of its rapid onset and relatively long-lasting anesthetic effect as compared to other commonly used LA [1]. Liposphere formulation of bupivacaine and lidocaine were previously evaluated as sustained release delivery system for LA [2]. Biostable systems such as hydro gels formed by different polymers and co-polymers have been used to release several drugs [3,4]. When the bioerodible system is used, one of the most important characteristics is its biocompatibility which means that the degradation products originated in the biodegradation process must be 
metabolized by the organism system based on polylactide and polyglycolide and their copolymer [5]. Chitosan is the common name of a linear random copolymer of $\beta-(1-4)$ linked -Dglucosamine whose molecular structure comprises a linear backbone linked through glycosidic bonds. The basic amine groups of this polysaccharide are protonated and thus positively charged in the most physiological fluids. Chitosan is mostly hydrophilic and the proportion of acetylated monomers and their distribution in the chains has a critical effect on its solubility and conformation in aqueous media. These characteristics of chitosan have attracted many scientists working in biomedical area and particularly in drug delivery [6-9]

Many structural factors such as concentration of polymeric matrix, pKa of the ionizable group, degree of ionization, cross linking, density, hydrophillicity influence the degree of swelling of polymers [10]. Since it can be biodegraded by enzyme action [11], the efficacy of conventional treatments of oral diseases i.e. Dental caries, fungal infections is often reduced by the limited retention of the applied formulation within the oral cavity [12-13]. The short residence time of the formulation at its intended site of action may result in reduced bioavailability [13].

A large number of studies have documented the use of buccal delivery system for control release of drugs [13]. Chitosan microspheres as a formulation tool for the enhanced retention of a therapeutic entity within oral mucosa have been evaluated by in vitro test. One of the greatest challenges for applying pharmaceutical formulation based on chitosan in oral cavity is the presence of lysozyme; an enzyme widely distributed in different mammalian fluids such as saliva or vaginal fluid [14-15]. The literature has described the hydrolytic activity of lysozyme on chitosan [15]. The aim of this research was to develop an easy and simple sustainable oral drug delivery system using bupivacaine loaded glutaraldehyde cross-linked high molecular weight chitosan microsphere for buccal administration.

\section{EXPERIMENTAL}

\section{Materials}

High molecular weight chitosan (HPC, mol wt $\{1.4-2.2\} \times 10^{5}$ ) (Sigma Aldrich, Belgium.), Glutaraldehyde (sd fine chemicals, India), lysozyme (Sigma Aldrich, China), polyglycolic acid (PGA) (SD Fine Chemicals, India), Methanol (SD Fine Chemicals, India), Bupivacaine (Fluka Analytical, Italy) were purchased. All other chemicals used were of analytical grades, and double-distilled water was used throughout the study.

\section{Preparation of bupivacaine-loaded chitosan beads}

Bupivacaine loaded chitosan beads were produced using the method reported elsewhere [15]. In brief, $2 \% \mathrm{w} / \mathrm{v}$ chitosan solution was prepared by dissolving medium weight chitosan in $2 \% \mathrm{v} / \mathrm{v}$ acetic acid overnight at room temperature under constant stirring. Then the resulting solution was filtered to remove any physical impurities and $100 \mathrm{mg}$ of bupivacaine was dissolved in $10 \mathrm{ml}$ of the filtrate. The resulting solution was mixed with sodium hydroxide solution as a coagulant and methanol followed by syringe injection to form beads; glutaraldehyde $(5 \mathrm{~mol} / \mathrm{L})$ was added as a cross linking agent and incubated for $4 \mathrm{~h}$ at $25^{\circ} \mathrm{C}$ to allow the beads to harden. Then beads were filtered and washed with excess solution of sodium bisulphate to remove excess of glutaraldehyde and finally beads were dried at room temperature.

\section{Preparation of bupivacaine-loaded films}

Bupivacaine/polyglycolic acid (PGA) films were prepared using the method reported elsewhere [15]. In brief, $50 \mathrm{mg}$ of PGA (50 mg) was dissolved in $5 \mathrm{ml}$ of chloroform; $1 \mathrm{ml}$ of resulting was spread on a glass plate. The resulting films were allowed to set at $27{ }^{\circ} \mathrm{C}$ for complete drying; $100 \mathrm{mg}$ of bupivacaine-loaded beads were dispersed on the film and subsequently $1 \mathrm{ml}$ of PGA solution was added. Chloroform was completely evaporated and the resulting film was dried at $27^{\circ} \mathrm{C}$ over calcium sulphate $\left(\mathrm{CaSO}_{4}\right)$ for $24 \mathrm{~h}$ and stored in $\mathrm{a} \mathrm{CaSO}_{4}$ desiccator.

\section{Determination of drug content and encapsulation efficiency}

The concentration of drug in all the formulations was determined by UV-visible-NIR spectrophotometer $670 \mathrm{~V}$ (Jasco, USA). Bupivacaine-loaded chitosan beads $(50 \mathrm{mg})$ were added to $57 \mathrm{ml}$ of phosphate buffer ( $\mathrm{pH} 7.5)$ containing $5 \mathrm{mg}$ of lysozyme followed by stirring for 5 days at $37^{\circ} \mathrm{C}$. Assay of bupivacaine content was done using spectrophotometer at $\lambda_{\max }$ of 263 $\mathrm{nm}$. Assay was performed in triplicate. The drug content (DC, \%) and encapsulation efficiency (EE, \%) were calculated as in Eqs 1 and 2.

$\mathrm{DC}=(\mathrm{Md} / \mathrm{Mm}) 100$ 
where $\mathrm{Md}$ is mass of drug in the microsphere and $\mathrm{Mm}$ is the mass of microsphere.

$E E=\left(D_{L} / T_{L}\right) 100$

where $D_{L}$ is actual drug loading and $T_{L}$ is theoretical drug loading.

\section{Swelling studies}

Swelling of PGA blended with chitosan microsphere prepared using three different concentrations of cross linker as well as three different drug loadings were studied in water by measuring mass uptake at $\mathrm{pH}$ 7.4. Accordingly, the prepared networks in the form of beads were tested by placing $30 \mathrm{~mL}$ buffer solution $(\mathrm{pH} 7.4)$ each and incubated at $60^{\circ} \mathrm{C}$. An appropriate time interval (30 min), beads were taken out and excess water was removed from the polymeric blends using filter paper and blends were weighed immediately using electronic weighing balance of accuracy $0.00001 \mathrm{mg}$ (Shimadzu, Japan). The swelling of microsphere was expressed as swelling ratio (S) using Eq 3.

$\mathrm{S}=\left(\mathrm{W}_{1}-\mathrm{W}_{0}\right) / \mathrm{W}_{0}$

where $W_{1}$ is the weight of the sample at time $t$ and $W_{0}$ is the weight of dry sample.

\section{X-ray diffraction studies (XRD) studies}

X-ray diffraction $(\mathrm{XRD})$ studies were carried to help to determine the crystallinity of the drug in the cross-linked network (Bucker Germany, CuKó radiation, Nickel filter). The XRD patterns of bupivacaine-loaded chitosan-PGA blend, blank bupivacaine loaded chitosan-PGA blend, and the pure drug were compared.

\section{Scanning electron microscopy (SEM) studies}

The surface and cross sectional morphologies of chitosan/citrate films were examined using high resolution scanning electron microscopy (HRSEM) (FEI Quanta FEG 200-high resolution scanning electron microscope).

\section{Differential scanning calorimetry (DSC)}

Pure bupivacaine and the drug-loaded microsphere were subjected to DSC (DSC Q 1000V9.4 Build 287) analysis at a heating rate of $10^{\circ} \mathrm{C} / \mathrm{min}$ over temperature range of $0-400^{\circ} \mathrm{C}$.

\section{In-vitro release study}

In vitro drug release studies were performed using a United States Pharmacopeia paddle-type dissolution apparatus (DA-1D, V Scientific, India) containing $100 \mathrm{ml}$ of phosphate buffer $(\mathrm{pH} 7.4)$ as the dissolution medium. The film $(\sim 50 \mathrm{mg})$ was used for the study enclosed in a dialysis bag. Dissolution studies were performed at $37 \pm$ $2{ }^{\circ} \mathrm{C}$ at $500 \mathrm{rpm}$. Samples $(5 \mathrm{ml})$ were withdrawn from the medium at various time intervals and the medium replenished immediately with the same volume of fresh dissolution medium to maintain sink conditions. The amount of dissolved drug in the sample taken was measured (after filtration through Whatman filter paper 41, pore size: $25 \mu \mathrm{m}$ ) spectrophotometrically at $\lambda_{\max }$ of $263 \mathrm{~nm}$; a parallel experiment was conducted in which $5 \mathrm{mg}$ of lysozyme was incorporated in the diffusion medium. Data obtained from in vitro release studies were fitted to Higuchi model to determine the mechanism of drug release from the chitosan microsphere. The tests were carried out in triplicate.

\section{Statistical analysis}

Statistical analysis was performed using Origin 7.0 Scientific graphing and analysis software. All the tests were run in triplicate and the drug release data analyzed by one-way ANOVA with statistical significance set at $p<0.05$.

\section{RESULTS}

\section{X-ray diffraction (XRD)}

XRD patterns (Fig 1) indicate that bupivacaine showed characteristic intense peaks at $10^{\circ}$ and $30^{\circ}$ due to the crystalline nature of the drug. However, peaks for the plain drug were not seen for the drug-loaded microspheres due to encapsulation of the drug in the polymeric network, and same applied to the blank microspheres, thus indicating that the encapsulated drug became amorphous in nature.

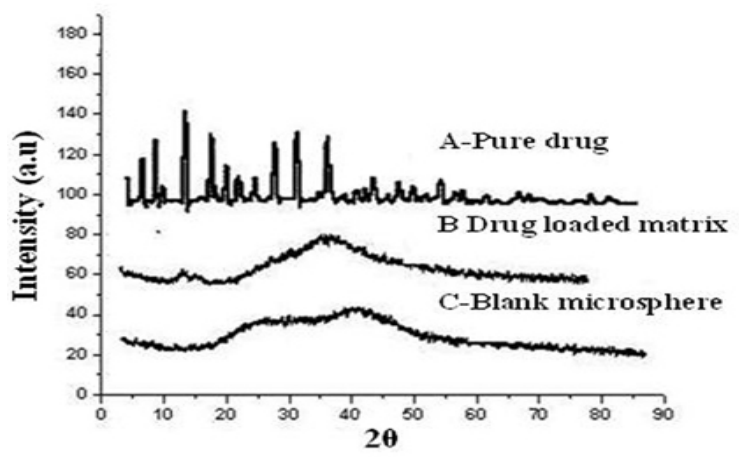

Fig 1: XRD diffractogram of $(A)$ pure drug (B) drugloaded chitosan matrix $(\mathrm{C})$ blank microspheres

\section{Scanning electron microscopy}

The surface morphology of the drug-loaded microspheres, shown in Fig 2A, indicates that 
they were fairly smooth and spherical in shape. Bupivacaine-loaded chitosan microspheres were embedded in PGA films but aggregated (Fig 2B). The results indicate good compatibility between the matrix and the drug.
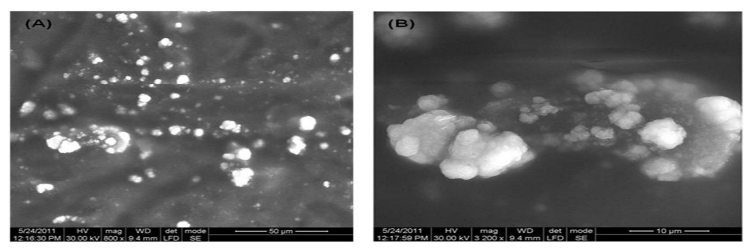

Fig. 2 (A) SEM of bupivacaine-loaded chitosan microspheres, (B) SEM of bupivacaine-loaded chitosan microsphere embedded in PGA

\section{Differential scanning calorimetry (DSC)}

DSC thermograms of chitosan, formulated microspheres and pure drug are shown in Figure 3. The thermograms of chitosan showed a broad peak at $109{ }^{\circ} \mathrm{C}$ which was attributed to loss of water due to evaporation of absorbed water and no degradation, which would normally occur at $280{ }^{\circ} \mathrm{C}$, was observed for chitosan in the thermograms, [17-18]. Bupivacaine thermograms showed a broad beak at $255{ }^{\circ} \mathrm{C}$ which corresponds to its melting point which is usually in the range of $255-256{ }^{\circ} \mathrm{C}$. This peak was absent in the thermograms for the drug-loaded chitosan microspheres, however, a peak appeared at $239{ }^{\circ} \mathrm{C}$. This means that chitosan probably conjugated with bupivacaine to form a stable adduct. The conjugation between drug and chitosan was broken on heating and hence a new peak appeared at $239^{\circ} \mathrm{C}$.

\section{Drug content and encapsulation efficiency}

Drug content and encapsulation results are shown in Table 1. Three different loading concentrations of bupivacaine were assessed during cross-linking of the microspheres. The results show the increasing trends with increasing drug loading. Encapsulation efficiency of $63 \%$ was observed for plain chitosan microsphere but it ranges were from $67.4 \pm 0.6 \%$ to $83 \pm 0.8 \%$ for the other formulations.

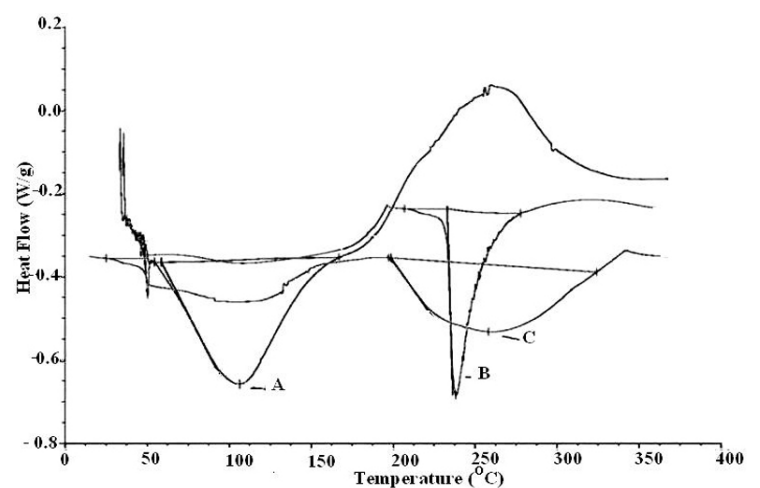

Fig 3: DSC thermograms of (A) chitosan, (B) chitosan-drug loaded microspheres, and $C$ ) pure drug

\section{In vitro drug release}

The release profile of bupivacaine from the chitosan beads is shown in Fig 4. In vitro results show that in the presence of lysozyme, $39 \%$ of the drug was released from the microspheres after 4.5 days while maximum drug release $(42.5 \%)$ was achieved on day 11 . This compares with $31 \%$ drug release on day 4.5 in the absence of lysozyme and,38 \% on day 11 . Maximum drug release was achieved on day 11 in the presence of lysozyme.

On subjecting the drug release data to Higuchi model $(Q=K t 1 / 2)$ in order to ascertain the drug release mechanism, a linear relationship was observed with a regression coefficient $\left(r^{2}\right)$ of 0.990 .

\section{DISCUSSION}

lonized chitosan hydro gel and its swelling characteristics are dependent on its chemical structure and $\mathrm{pH}$ of the medium. The amount of crosslinking agent added to the polymeric matrix at equilibrium was based on the concentration of the functional group present in the polymeric

Table 1: Encapsulation efficiency and swelling of the preparations

\begin{tabular}{|c|c|c|c|c|c|}
\hline Formulation core & $\begin{array}{l}\text { \%PGA } \\
\text { microphere }\end{array}$ & Bupivacaine (\%) & $\begin{array}{l}\text { Amount of } \mathrm{GA} \\
(\mathrm{ml})\end{array}$ & $\begin{array}{l}\text { Encapsulation } \\
\text { efficiency (\%) }\end{array}$ & $\begin{array}{l}\text { Swelling } \\
(\%) \pm S D\end{array}$ \\
\hline CS-PGA-1 & 10 & 5 & 2.5 & $71.1 \pm 0.3$ & $327 \pm 0.1$ \\
\hline CS-PGA-2 & 10 & 5 & 5 & $67.4 \pm 0.6$ & $302 \pm 0.6$ \\
\hline CS-PGA-3 & 10 & 5 & 7.5 & $62.7 \pm 0.2$ & $291 \pm 0.3$ \\
\hline CS-PGA-4 & 20 & 5 & 5 & $78.8 \pm 0.1$ & $369 \pm 0.6$ \\
\hline CS-PGA-5 & 30 & 5 & 5 & $83.1 \pm 0.8$ & $393 \pm 0.2$ \\
\hline CS-PGA-6 & 10 & 5 & 5 & $64.2 \pm 0.2$ & $278 \pm 0.3$ \\
\hline
\end{tabular}




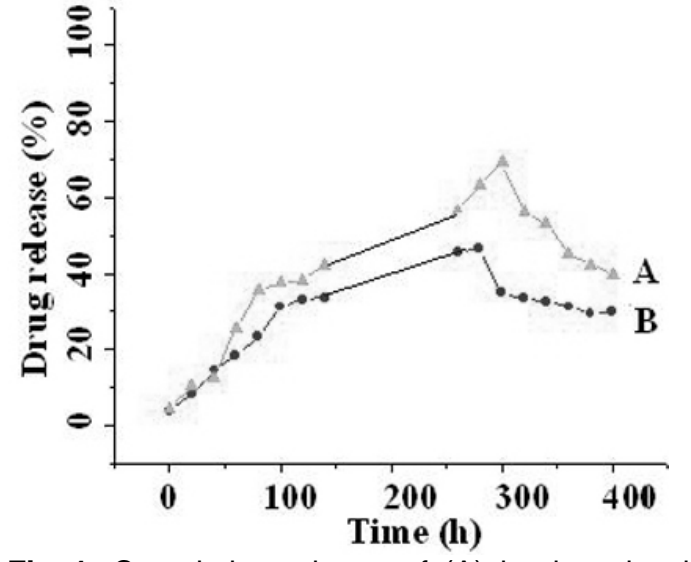

Fig 4: Cumulative release of $(A)$ bupivacaine in the presence of lysozyme, and (B) in the absence of lysozyme

network. The results of the present study show that the encapsulation efficiency was decreased with increased concentration of crosslinking agent glutaraldehyde from $71.1 \pm 0.3(\%)$ to $62.7 \pm 0.2$ and swelling from $327 \pm 0.1 \%$ to $291 \pm 0.3$ when the amount of PGA in microsphere (\%) and drug (\%) were same, but the encapsulation efficiency was increased with increased concentration of PGA from $64.2 \pm 0.2$ (\%) to $83.1 \pm 0.8$ and swelling from $369 \pm 0.5(\%)$ to $278 \pm 0.3$, when the amount of glutaraldehyde (\%) and drug (\%) were same. The strength and water preservation efficiency of the hydro gel depends on the amount of crosslinking agent used because the chitosan molecules can be transformed into polymeric network structure by the addition of crosslinking agent and consequently, water molecules can be preserved in the network developed. If amount of cross linking is less, this would greatly affect the strength as well as the water preservation efficiency of the hydro gel towards the lower end. If excessive amount of crosslinking agent is used, this would result in a decrease in network volume for water preservation efficiency [19].

The use of glutaraldehyde in the formulation of poorly soluble chitosan derivatives makes microspheres stronger [16]. Encapsulation efficiency increased with increasing PGA in the microspheres. This is due to the hydrophilic nature of PGA. Encapsulation efficiency decreased as the concentration of crosslinker increased. Such decreasing trend is due to increasing crosslink density because the microspheres became rigid, thereby reducing the free volume space within the polymer matrix and hence a reduction in encapsulation efficiency.

The extent of crosslinking was dependent upon equilibrium swelling. For instance, equilibrium swelling ratio decreased from $327(\%)$ to $291(\%)$ leading to increasing crosslinking density and decreased pore volume of the formulation matrix. The XRD and DSC results obtained indicated that there were no interactions between bupivacaine, chitosan and other ingredients used in preparing the microsphere. This implies that the developed microspheres would be stable during the course of drug delivery. have disappeared in the drug loaded chitosan microspheres, it suggests the lowering of crystallinity in drug loaded matrix and results in more dispersion the polymeric matrix which indicates higher release in the system. Similar type of findings was reported when an amorphous drug, which is very difficult to measure at the detection limit of the crystal size, was dispersed molecularly in the polymer matrix and hence, no crystals were found in the drug loaded matrices [20].

Chitosan microparticles may owe their promising mucoadhesive performance to their cationic nature. It has been suggested that within aqueous environment cationic materials display a mechanism of mucoadhesion in which not only hydrogen bonds, but also salt bridge effects involving the positively charged chitosan microparticles and the negatively charged mucous glycoproteins. The chitosan formulation in oral cavity releases more drug in the presence of lysozyme, an enzyme widely distributed in mammalian fluids such as saliva and vaginal fluid in cells like macrophages [14]. The hydrolytic activity of lysozyme on chitosan has demonstrated that the presence of lysozyme increases the total amount of drug released from the microsphere.

\section{CONCLUSION}

Bupivacaine-loaded microspheres have been successfully prepared by emulsification technique, and the formulation seemed suitably designed for the control of dental pain in the buccal cavity, where lysozyme would act by facilitating bupivacaine release. Controlled release of bupivacaine in vitro up to 11 days can be achieved. Thus, high molecular weight chitosan microsphere embedded in PGA film may be suitable for in vivo delivery of bupivacaine in buccal cavity. Further research on this formulation is, however, required.

\section{REFERENCES}

1. Physicians' Desk Reference, 57th Edition. Montvale, NJ. Medical Economics, 2003, 642-644.

2. Malinovsky JM, Bernard JM, Le Corre $P$, Dumand JB, Lepage JY, Le Verge $R$, Souron $R$. Motor and Blood pressure effects of epidural sustainedrelease bupivacaine from polymer microsphere: a 
dose response study in rabbits. Anesth Analg 1995; 81: 519-524.

3. Blanco MD, Trigo RM, Teijon C, Gomez C, Teijon JM. Slow releasing of ara- $C$ from poly (2-hydroxyethyl methacrylate-co- $N$-vinyl-2-pyrrolidone) hydro gels implanted subcutaneously in the back of rates. Biomaterials 1998; 19: 861-869.

4. Gomez C, Blanco MD, Bernardo MV, Sastre RL, Teijon JM. Poly(acryl amide-co-monoethyl itaconate) hydro gels as devices for cytarabine release in rats. J Pharm Pharmaco 1998; 50: 703-712.

5. Le Corre P, Rytting JH, Gajan V, Chevanne F, Le Verge $R$. In vitro controlled release of local anaestics from poly $(D, L$-lactide $)$ microspheres. Microencapsulation 1997; 14: 243-255.

6. Dutta PK, Dutta J, Tripathi VS. Chitin and Chitosan: Chemistry, properties and application. J Sci Ind Res 2004: 63: 20-31.

7. Csada N, Garcia-Fuentes M, Alonso MJ. The performance of nano carriers for transmucosal drug delivery. Expert Opin Drug Deliv 2006; 3: 463-478.

8. Csada N, Garcia-Fuentes, M, Alonso MJ. Nanoparticles for nasal vaccination. Adv Drug Deliv Rev 2009; 61. 140-157.

9. Garcia-Fuentes M, Alonso MJ. Chitosan-based drug nano carriers: Where do we stand?

Rev J Cont Release 2012; 161: 496-504.

10. Baras B, Benoit MA, Gillard J. Influence of various technological parameters on the preparation of spray-dried poly(E-caprolactone) microspheres containing a model antigen. J Microencapsul. 2000; 17: 485-498.

11. Muzarelli RAA, Biagini G, DeBenedittis A, Mengucci $P$, Majni G, Tosi G. Chitosan-oxychitin coatings for prosthetics materials. Carbohyd Polymers 2001, 45: 35-41.

12. Kockisch S, Rees GD, Young SA, Tsibouklis J, Smart JD. Polymeric microspheres for drug delivery to the oral cavity: An in vitro evolution of mucoadhesive potential. J Pharm Sci 2003; 92: 1614-1623.

13. Diaz del Consuelo I, Falson F, Guy RH, Jacques Y. Ex vivo evalution of bioadhesive films for buccal delivery of fentanyl. J Control Release 2007; 122: 135-140.

14. Bernardo MV, Blanco MD, Sastre RL, Teijon C, Teijon $J M$. Sustained release of bupivacaine from devices based on chitosan. IL Farmaco 2003; 58: 11871191.

15. Colfen H, Harding SE, Varum KM, Winzor DJ. A study by analytical ultracentrifugation on the interaction between lysozyme and extensively deacetylated chitin (chitosan). Carbohyd Polymers 1996; 30: 4563.

16. Coffin M, Parr A: US Patent 5407 687, April 18, 1995.

17. Dhanikula $A B$, Panchagula $R$. Development and characterization of biodegradable chitosan films for local delivery of paclitaxel. AAPS J 2004; 27: 1-5.

18. Cervera MF, Heinamaki J, Krogars $K$, Jorgenson AC. Solid state and mechanical properties of aqueous chitosan-Amylose starch films plasticized with polyols. AAPS Pharm Sci Tech 2004: 15: 1-6.

19.Wu J, Lin J, Wei C, Li G. Influence of the $\mathrm{COOH}$ and COONa groups and crosslink density of poly(acrylic acid)/montmorillonite superabsorbent composite on water absorbency. Polym Int 2001; 50: 1050-1053.

20 Ajit PR, Namdev BS, Sangamesh AP, Tejraj MA. Novel interpenetrating polymer network microspheres of chitosan and methylcellulose for controlled release of theophylline. Carbohyd Polym 2007; 69: 678687. 